\title{
3D nanomechanical evaluations of dermal structures in skin
}

Alexander P Kao ${ }^{1}$, John T Connelly ${ }^{2}$, Asa H Barber ${ }^{1,3 *}$

1 Department of Materials, School of Engineering \& Materials Science, Queen Mary University of London, London E1 4NS, UK

2 Centre for Cell Biology and Cutaneous Research, Barts and the London School of Medicine and Dentistry, Queen Mary University of London, E1 2AT, UK

${ }^{3}$ School of Engineering, University of Portsmouth, Portsmouth P01 2UP, UK

\section{*Corresponding Author}

asa.barber@port.ac.uk

+44(0)2392842363

\section{Keywords}

Skin, AFM, nanomechanics

\section{Highlights}

AFM techniques were used to measure the anatomical location dependent nanomechanics of skin in three dimensions.

A mechanically non-evasive freezing and thawing preparation method was found to retain nanomechanical behaviour of the skin, thus enabling mechanical investigations of skin sections.

Isotropic response of the elastic modulus in dermis at the nanoscale was demonstrated.

\section{Abstract}

Skin is a multilayered multiscale composite material with a range of mechanical and biochemical functions. The mechanical properties of dermis are important to understand in order to improve and compare on-going in vitro experiments to physiological conditions, especially as the mechanical properties of the dermis can play a crucial role in determining cell behaviour. Spatial and isotropy variations in dermal mechanics are thus critical in such understanding of complex skin structures. Atomic force microscopy (AFM) based indentation was used in this study to quantify the three dimensional mechanical properties of skin at nanoscale resolution over micrometer 
length scales. A range of preparation methods was examined and a mechanically nonevasive freeze sectioning followed by thawing method was found to be suitable for the AFM studies. Subsequent mechanical evaluations established macroscale isotropy of the dermis with the ground substance of the dermis dominating the mechanical response. Mechanical analysis was extended to show significant variation in the elastic modulus of the dermis between anatomical locations that suggest changes in the physiological environment influence local mechanical properties. Our results highlight dependence between an isotropic mechanical response of the dermal microenvironment at the nanoscale and anatomical location that define the variable mechanical behaviour of the dermis.

\section{Introduction}

Skin is a complex biological composite material that performs a variety of functions including serving as a barrier to the external environment and transferring mechanical loads. The complexity of skin is of continuing interest particularly for developing synthetic skin equivalents (Grover et al., 2012; Wang et al., 2012) as well as from more fundamental studies into understanding the biological optimization used to give flexibility and toughness (Groves et al., 2013). Anisotropic fibrous components at relatively small length scales within skin are of significant interest as their organization is critical in defining potentially complex mechanical anisotropy within bulk skin (Gahagnon et al., 2012; Gerhardt et al., 2012; Groves et al., 2013; Ní Annaidh et al., 2012b). The relationship between resultant mechanical performance, structural development and resultant organization within skin has been identified as a considerable challenge due to this mechanical complexity that exists (Delalleau et al., 
2006). Skin is structured as a multicomponent composite, as found in many biological materials with a mechanical function, while providing an environment for cells to grow and replenish the skin. From a structural mechanics approach, skin is a layered composite consisting of the dermis and the epidermis (Silver et al., 2003). The dermis makes up a higher volume fraction of the skin compared to the epidermis, and is mainly comprised of collagen fibres, elastic fibres, and ground substance of proteoglycans and glycoproteins (Silver et al., 2001). The fibrous collagen forms the main structural component of the dermis while the ground substance is an amorphous matrix that surrounds the collagen. These components comprise the dermal extracellular matrix (ECM) that dermal fibroblasts occupy and maintain.

The complex three dimensional (3D) microenvironment that fibroblasts are exposed to within the dermis is key to understanding the behaviour and activity of the cells (Lo et al., 2000; Rehfeldt et al., 2007; Saez et al., 2005; Solon et al., 2007). Mesenchymal stem cell fate has also been shown to be directly influenced by the mechanical environment used to culture stem cells (Engler et al., 2006). The stiffness of the microenvironment or ECM is therefore critical in determining the behaviour of the cell and is expected to vary according to the 3D organization found in the dermis.

Attempts to understand a macroscopic mechanical environment have led to studies investigating the mechanical properties of whole skin in response to various loading conditions. These macroscopic tests often utilise various experimental techniques including tension, compression and suction to establish the elastic modulus, yield strength and ultimate tensile strength of whole skin. Testing of whole skin indicates the resultant elastic modulus lies between $5.5 \mathrm{kPa}$ and $83.3 \mathrm{MPa}$, depending on the evaluation method used (Diridollou et al., 2000; Gahagnon et al., 2012; Gąsior- 
Głogowska et al., 2013; Geerligs et al., 2011; Groves et al., 2013; Hendriks et al., 2003; Jachowicz et al., 2007; Ottenio et al., 2014; Wang et al., 2013; Yuan and Verma, 2006; Zahouani et al., 2009). Uniaxial tensile testing of whole skin shows a dependence of the elastic modulus to the direction of the applied load and the alignment of the collagen fibre network of the dermis in the sample, indicating some degree of anisotropy in the dermis (Gąsior-Głogowska et al., 2013; Ní Annaidh et al., 2012b). These macroscopic tests provide insight into the bulk properties of skin in response to extreme loading conditions, but do not give any indication of the microenvironment within the bulk material that cells are exposed to. Therefore, reducing the length scale examined provides some measure of mechanical properties relevant to the loading conditions cells are exposed to. Measurements of the stiffness of tissues have been carried out using atomic force microscopy (AFM) to examine the mechanical properties at small length scales (Crichton et al., 2013; Last et al., 2009). AFM allows for mechanical characterisation at the nanoscale and can provide high spatial resolution of the surface properties of the material. Many biological materials have been examined by AFM in order to determine the mechanical properties (Hang and Barber, 2011; Hang et al., 2014), including AFM indentation of the dermis (Grant et al., 2012). Such an approach has provided a 2D mechanical map through a thickness cross section of skin or upper dermal surface after removal of the epidermal layers. These studies have provided mechanical information by reporting the elastic modulus of the probed area. The range of reported elastic modulus varied from $0.77 \mathrm{kPa}$ to $322 \mathrm{kPa}$ for human dermal tissue and $6.43 \mathrm{kPa}$ to $11.62 \mathrm{MPa}$ for mouse ear dermal tissue (Achterberg et al., 2014; Crichton et al., 2011; Grant et al., 2012; Petrie et al., 2012). Crichton et al. characterised the distribution of collagen fibres within the probed area indicating the contribution of collagen fibres within the region to the measured elastic modulus (Crichton et al., 2011). 
AFM indentation of upper dermal tissue from a human donor to depths of hundreds of nanometres resulted in a measured elastic modulus of $322 \mathrm{kPa}$, and also reported higher elastic modulus values in the megapascal range potentially influenced by collagen fibres in the probed region (Grant et al., 2012). Previous studies have commented on the possible contribution of the collagen fibres to the elastic modulus of the dermis, with the elastic modulus of collagen fibres reported by indentation being 1 2 GPa (Heim et al., 2006), where potential contact of indenter with the collagen fibre bundles increasing the measured elastic modulus of the dermis (Grant et al., 2012).

The importance of the directionality of the collagen network within the dermis on resultant skin mechanics have led to extensive experimental and numerical modelling methods. Uniaxial testing of human back skin excised from different orientations of the Langer's lines showed significant differences in the measured elastic modulus, with samples tested parallel to the Langer's lines showing a higher elastic modulus than samples tested perpendicular to the lines (Ní Annaidh et al., 2012b; Ottenio et al., 2014). Tensile testing was also modelled using finite element methods to produce a numerical model to predict the anisotropic behaviour of human and murine skin (Groves et al., 2013; Ní Annaidh et al., 2012a). Numerical models showed significant contribution of the alignment of the collagen fibres within the dermis to the mechanical behaviour of skin. These experimental and numerical results consider macroscopic scale testing and applying a large strain to skin with the assumption that the collagen fibres will be exposed to the loading conditions. However, no studies currently report on the effect of the direction of the applied load on the mechanical properties of the dermis at the nanoscale, where directionality could have an effect on the microenvironment experienced by cells within the dermis. This work aims to explore the 3D mechanics of 
the dermis by utilising AFM indentation in conjunction with a range of tissue preparation methods to establish a comprehensive understanding of the elastic behaviour of the dermis through relationships between directional nanoscale mechanical measurements and macroscopic mechanical properties. A particular focus is on evaluating the spatial 3D mechanical microenvironment in order to understand the response of the tissue to the directionality of the loading conditions.

\section{Methods}

Preparation of dermal explants

Mouse skin samples were taken from freshly excised wild type C57BL/6 mouse tails and were prepared as described by Braun et al. (Braun et al., 2003). Briefly, mouse tails were harvested and the skin cut lengthwise along the tail down to the point where contact was made with the bone. Animals were sacrificed according to Home Office regulations (license PPL 70/7166). Skin was peeled away from the tail using forceps to isolate the full skin thickness and allow further sample preparation. The bulk skin sample was sectioned into smaller samples with a plan surface of approximately $0.5 \mathrm{x}$ $0.5 \mathrm{~cm}$ in size. The sectioned skin samples were incubated in $0.005 \mathrm{M}$ Ethylenediaminetetraacetic acid (EDTA) at $37{ }^{\circ} \mathrm{C}$ for approximately four hours. The EDTA solution acted as a chelation agent that disrupted the cation mediated joining of the epidermal layers and promoted separation of the epidermis from the dermis. The epidermis was peeled away from the dermis using forceps, rinsed with PBS to remove any excess EDTA that may be still present on either the epidermis or the dermis and then stored in a PBS solution with $0.2 \%$ sodium azide and $0.05 \%$ protease inhibitor. The 
process of removing the epidermis was conducted while the skin samples were submerged in PBS to prevent any dehydration of the tissue. Sodium azide was added to the solution in order to prevent infection or the growth of bacteria, and the addition protease inhibitor slowed down the degradation of the extra cellular matrix. The sections of dermis were stored at $4{ }^{\circ} \mathrm{C}$ until use. Three mouse tails were used in total and 11 dermal explant samples were produced for analysis. The dermal explants produced by the mechanical peeling process do not have any definable surface features that can be used to accurately relocate a specific location on the sample surface. The position of the AFM measurements is critical for assessing any changes in the mechanical behaviour on the surface of the dermal explants and required a method for relocating a previously measured area of the sample. A coordinate system was established by patterning a micron scale grid onto the sample surface using a TEM grid (Agar Scientific, UK) as a negative lithography pattern. The spacing between the lines of the TEM grid provides features that are well defined and evenly spaced. The patterning process involved first attaching the TEM grid to the dermal surface by mechanical clips, with surface water removed from the sample using laboratory wipes. Waterproof ink was then deposited over the area of the sample covered by the grid, followed by grid removal from the sample to give a micron scale grid pattern on the surface of the dermal explant. Care was taken to complete the patterning of the samples before any dehydration of the tissue occurred due to evaporation. The patterns produced using this method are robust enough to remain visible and well defined throughout the imaging and measurement process in a liquid environment, as well as after the freezing and thawing process. The patterning process is schematically shown in Figure 1 along with the resulting grid as observed by optical microscopy. 


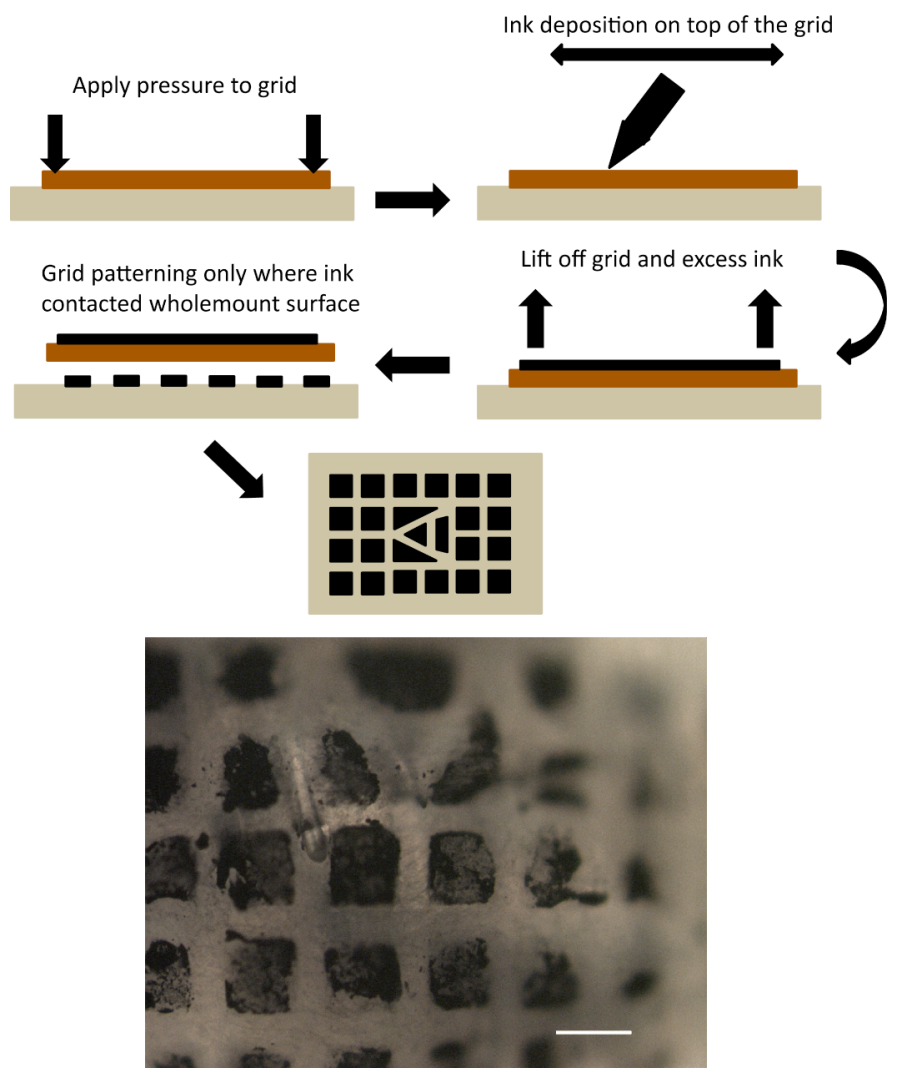

Figure 1. (Top) Schematic representation of the patterning process applied to the dermal explant samples. (Bottom) Optical microscopy image of a patterned dermal explant, scale bar $50 \mu \mathrm{m}$.

Procedure for freeze-thaw cycling

Dermal samples prepared and patterned as outlined above were used to test a preparation method suitable for subsequent small scale mechanical testing while retaining the inherent mechanical properties of the skin. A cryoprotective agent consisted of a $1 \mathrm{M}$ solution of sucrose with $10 \% \mathrm{v} / \mathrm{v}$ of DMSO was used to condition the skin prior to freezing and evolution of surfaces for AFM indentation by sectioning. DMSO and sugar solutions are common cryoprotective agents that are used to preserve cellular viability when freezing cells and tissue, such as skin, arteries, and tendons, to 
cryogenic temperatures (Bakhach, 2009; Bravo et al., 2000; Chen et al., 2011; Fuller, 2004) and are thus explored here. Dermal samples were first placed in 10\% v/v DMSO in PBS at $4{ }^{\circ} \mathrm{C}$ in $1 \mathrm{~mL}$ tubes until the sample reached the bottom of the tube. Samples were then transfer to $1 \mathrm{M}$ sucrose with $10 \% \mathrm{v} / \mathrm{v}$ DMSO at $4{ }^{\circ} \mathrm{C}$ until the sample again reached the bottom of the tube. Tubes containing the sample and CPA were then placed in a $-80{ }^{\circ} \mathrm{C}$ freezer overnight. Tubes were removed from the freezer, allowed to return to room temperature and transferred into $10 \% \mathrm{v} / \mathrm{v}$ DMSO. Samples were rinsed several times in PBS in order to ensure removal of CPA and stored in PBS at $4{ }^{\circ} \mathrm{C}$ until use. Four dermal explant samples were treated with the CPA and three were left untreated.

\section{Analysis of freeze thaw cycling}

Patterned dermal explant samples exhibited micron sized features resolved using optical microscopy and used to accurately reposition the AFM probe tip at a specific location on the sample surface for subsequent mechanical evaluations. $20 \mu \mathrm{m} \times 20 \mu \mathrm{m}$ areas were selected on the dermal samples in order to perform AFM indentation to map the distribution of elastic modulus values. A grid of $20 \times 20$ AFM indentations was performed within the selected area. Repeat measurements of the entire area were conducted prior to freezing of samples to establish a baseline variation in the elastic modulus measurement of a single point. This step was taken in an attempt to limit the experimental error in repositioning the AFM probe after the sample was treated. The baseline data sets were first reduced from a $20 \times 20$ matrix of 400 points to a $10 \times 10$ matrix of 100 points by averaging over four adjacent indentation values. The mechanics of the dermal surface should not vary significantly within the $2 \times 2$ area so a local average of each data point also allows for a better representation of the elastic modulus for a specific region of the selected area. No changes to the experimental setup with 
regards to the position of the sample or probe tip were used in order to maintain measurement consistency. The variation between the repeat measurements from before and after the freeze-thaw cycle was quantified by calculating the root mean square (RMS) of the difference at each spatial point within the averaged $10 \times 10$ matrix of elastic modulus values of the measured area using the following equation:

$$
R M S=\sqrt{\frac{\sum_{1}^{i}\left(E_{N, i}{ }^{2}\right)}{n}}
$$

where $E_{N, i}$ is the difference between the first set of measurements before the freezethaw cycle and the second data set after the freeze-thaw cycle at each measured point, $i$. The data sets were normalised against the largest elastic modulus value within the pair prior to calculating the difference at each point within the array. The calculated RMS of the data sets using Equation (1) allowed for a comparison between each data point before and after the freeze-thaw cycle using a single parameter to evaluate the magnitude of the change. Thus, for example, two identical elastic modulus data sets from before and after the freeze-thaw cycle would give a resultant RMS zero value whereas larger differences in the before and after data sets would give increasing RMS values. A larger RMS value is physically important and denotes artefacts from the sample preparation that cause a modification to the mechanical properties of the skin.

\section{Preparation of transverse sections of skin}

AFM indentation analysis was performed on transverse cryosections of mice tails, obtained from C57BL/6 mice between the ages of 7 and 9 weeks, in order to examine the through thickness mechanics of the dermis. The whole skin was peeled away from 
the tail and cut into $0.5 \mathrm{~cm} \times 0.5 \mathrm{~cm}$ sections. Samples were then embedded in OCT (Tissue-tek Sakura, EU), frozen over dry ice and transferred to a freezer at $-80{ }^{\circ} \mathrm{C}$ prior to sectioning. Transverse sections were cut using a Leica CM1860 cryostat (Leica Biosystems, UK) using a cutting temperature of $-20{ }^{\circ} \mathrm{C}$. Whole skin sections were cut to $5 \mu \mathrm{m}$ in thickness and transferred to glass slides (Superfrost Plus, Thermoscientific, UK) and stored at $-80^{\circ} \mathrm{C}$ prior to thawing and subsequent mechanical testing. Three mouse tails were cryosectioned and one section from each mouse was analysed using AFM indentation.

\section{AFM analysis}

AFM measurements were conducted using standard equipment (Ntegra AFM system, NT-MDT, Russia) with a sample stage mounted on piezoelectric positioners. In situ optical microscopy was used to position the AFM probe away from any remaining hair follicles on the dermal surface. The dermal explant measurements were carried out by submerging the samples in PBS at room temperature in a stainless steel liquid cell with an approximate volume of $1 \mathrm{~mL}$. The dermal samples were mechanically coupled to the bottom of the liquid cell, and the liquid cell was magnetically attached to the AFM positioners. Dermal samples were imaged in contact mode using a low cantilever set point value. AFM height images were taken over a $20 \mu \mathrm{m}$ × $20 \mu \mathrm{m}$ area. AFM indentation measurements were conducted using silicon nitride v-shaped cantilevers with a nominal spring constant between $0.01 \mathrm{~N} \cdot \mathrm{m}^{-1}$ and $0.5 \mathrm{~N} \cdot \mathrm{m}^{-1}$ (Bruker, USA). Each cantilever spring constant was calibrated using the thermal noise method (Butt and Jaschke, 1995) prior to all AFM measurements. The elastic modulus of the dermal explants was measured by AFM indentation using the force-displacement functionality. Force-displacement curves were recorded by translating the sample in the z-direction 
towards the AFM probe using the z-piezo positioner of the AFM. Subsequent contact and indentation of the AFM probe into the sample caused a corresponding cantilever deflection with z-piezo displacement as shown in Figure 2a.

The force-displacement curves were recorded at $1 \mu \mathrm{m}$ spacing intervals over a $20 \mu \mathrm{m} \mathrm{x}$ $20 \mu \mathrm{m}$ area to give a grid of indentations. Following all indentation measurements, calibration force-displacement curves were obtained on a sapphire sheet in order to determine the response of the photodiode to the cantilever deflection and determine the ratio of photodiode current $(\mathrm{nA})$ to cantilever deflection $(\mathrm{nm})$. The contact point of the force-displacement curves was determined using a custom script (MS Excel, USA) based on the algorithms of Lin et al. (Lin et al., 2007). The force-displacement curves showed elastic deformation indicated by the retracing of the force loading curve by the unloading curve. Probe sample adhesion behaviour was not observed and did not influence the analysis of the force-displacement curves. The absence of adhesion forces indicates that the contact between the AFM probe and the sample surface can be modelled as a Hertzian contact, simplifying the mechanical analysis. Force-displacement curves that did not have a clear contact point, characterized by a lack in observable change from the linear approach to the non-linear indentation portion of the curve, were excluded from the analysis, approximately $7.6 \%$ of the total measurements were rejected due to indistinct contact within the force-displacement curve.

The cantilever deflection current (nA), recorded from the AFM photodiode detector to quantify deflection, was transformed to physical cantilever deflection (nm) using the calibration ratio of deflection current to cantilever deflection obtained from performing indentations on a sapphire sheet as shown in Supplementary Figure 1. The total indentation depth was calculated by subtracting the cantilever deflection from the total 
displacement of the z-piezo positioner. The cantilever deflection was finally converted to force using Hooke's law, $F=k d$, where $k$ is the cantilever spring constant calculated using the thermal noise method (Butt and Jaschke, 1995) and $d$ is the cantilever deflection measured from the optical photodiode sensor in the AFM system. The applied force was plotted against the indentation depth to describe the interaction between the AFM probe and the sample surface. The AFM probe was modelled as a cone so that the indentation depth causes a corresponding increase in the area of the sample interacting with the probe. The resultant measured contact stiffness and applied force thus increased non-linearly with indentation depth. Such a non-linear relationship provides the elastic modulus of the sample surface by fitting the unloading portion of the forcedisplacement plot using the Oliver-Pharr method with Sneddon's approximation for a rigid cone indenter (Sneddon, 1948). The force applied by the indenter is related to the elastic modulus of the sample through the indentation depth, $d$, using:

$$
F=\frac{2 E}{\left(1-v_{s}^{2}\right) \pi} \tan \alpha \cdot d^{2}
$$

The force-distance curve shown in Figure $2 \mathrm{~b}$ was fitted to equation (2) to give the sample's elastic modulus by assuming a basic Hertzian linear elastic response of the material, a Poisson's ratio $v_{s}$ of 0.5 , a value used as soft biological samples are generally treated as incompressible (Domke and Radmacher, 1998; Grant et al., 2012; Radmacher, 1997), and a half tip opening angle $\alpha=17.5^{\circ}$ taken from the manufacturer's specifications and verified by scanning electron microscopy. Analysis and curve fitting to extract the elastic modulus from the force-displacement curves was conducted using a custom script (MS excel, USA). Mapping on dermal surfaces contained a minimum of 200 force displacement curves per sample with 11 dermal explant samples from three 
mouse tails used. Force-displacement indentations were performed on agarose gels of known elastic modulus to validate the curve fitting process, see supplementary information for details.

Force-displacement measurements were additionally conducted on the transverse sections of full thickness skin to investigate the effect of the orientation of the applied indentation load on the elastic modulus. The glass slides with skin sections were mounted to a magnetic sample holder using double sided tape. AFM measurements of transverse sections were conducted in a droplet of PBS at room temperature in order to keep the sample hydrated. Low set point AFM contact imaging was performed in order to locate the edge of the epidermis region within the section for identification of a reference structural feature in the skin. Force-displacement curves were obtained and analysed in the method described above. Force-displacement curves were taken at a spacing of $100 \mathrm{~nm}$ in the $\mathrm{y}$-axis of the sample and at a spacing of $500 \mathrm{~nm}$ in the $\mathrm{x}$-axis. A smaller y-axis spacing was employed here due to higher structural variation in this $y$ axis, extending through thickness across the layered features of skin, compared to the xaxis. The force-displacement curves were taken using a raster scan method allowing for correlation of each indentation point to the spatial $x-y$ position on the sample surface. The elastic modulus values of the transverse skin sections were then reconstructed into a 3D distribution map using Origin 8 (OriginLabs, USA) with elastic modulus values as z coordinates and the spatial location of the indentation as $x-y$ coordinates. Each transverse section area measured was at least $30 \mu \mathrm{m}$ in the $\mathrm{y}$ direction and $5 \mu \mathrm{m}$ in the $\mathrm{x}$ direction in order to ensure a region of the dermis was present within the examined section. The characteristic indentation depth was approximately $200 \mathrm{~nm}$ with the indentation depth limited to a maximum of $500 \mathrm{~nm}$ for the $5 \mu \mathrm{m}$ thick transverse cross 
section samples to avoid the influence of the glass substrate on the measurement of the elastic modulus, as defined from the limits of indentation proposed by previous work (Gavara and Chadwick, 2012). Indentations beyond $200 \mathrm{~nm}$ on the cryosectioned samples would result in overlap of the indentations in the y direction but would not alter the mechanical response as force-displacement curves indicate elastic behaviour. Additional transverse sections were taken from regions of each tail sample near to the area analysed by AFM to determine the thickness of the epidermis by haematoxylin and eosin histological staining. Cryosections from three mouse tails were analysed using AFM indentation.

\section{Results and Discussion}

Elastic modulus 2D maps for the dermal surface were recorded using AFM nanoindentation, with an example colour coded elastic modulus distribution map displayed in Figure 2c. The dermal surface refers to the sample surface after removal of the epidermis with the lamina densa of the basement membrane exposed, which has an approximate thickness of $40 \mathrm{~nm}$ (Osawa et al., 2003). The thickness of the basement membrane is significantly less than the indentation depth $(\sim 400 \mathrm{~nm})$, therefore the contribution to the mechanical response is expected to be less than $1 \%$ assuming the indentation senses up to 10 times the indentation depth (Tsui and Pharr, 1999). All AFM indentation data was analysed by correcting the raw deflection - z-piezo movement data, Figure 2a, to a force-displacement curve, Figure $2 \mathrm{~b}$, and analysing the forcedisplacement curve by fitting equation (2) for the elastic modulus. The distribution maps show a range of elastic moduli within the measured area indicating heterogeneous mechanics across the surface of the dermis. Such variability in dermis 
mechanical properties is expected due to the potential for indentation of constituent components within the dermis. Indentation of collagen fibre bundles are expected to produce a higher measured elastic modulus value when compared to indentation of the dermal ground substance, with the ground substance being more of a gel-like matrix surrounding the high elastic modulus collagen fibre bundles (Zahouani et al., 2009). The range of raw elastic moduli for the dermis falls between a minimum value of 0.148 $\mathrm{kPa}$ and a maximum value of $9.77 \mathrm{MPa}$ with the mean value of the distribution appearing at $137 \pm 6.28$ (Standard error of mean, SEM) $\mathrm{kPa}$ and a median value of 44.3 $\mathrm{kPa}$. The large range in elastic modulus for the dermis samples represents the complex multicomponent system where varying surface composition influences the elastic modulus measured by the AFM indentation.

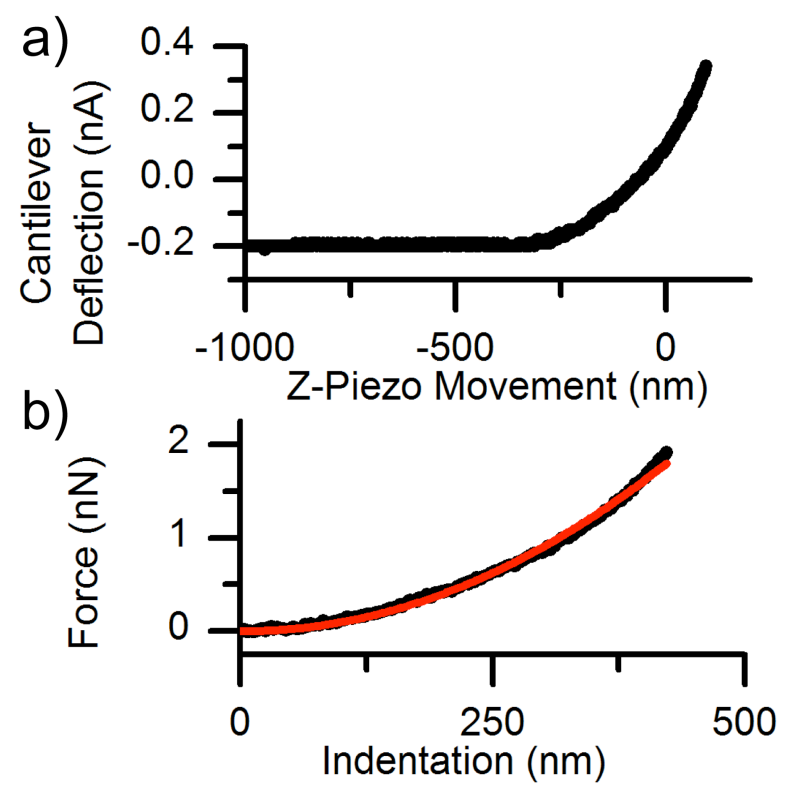

c)

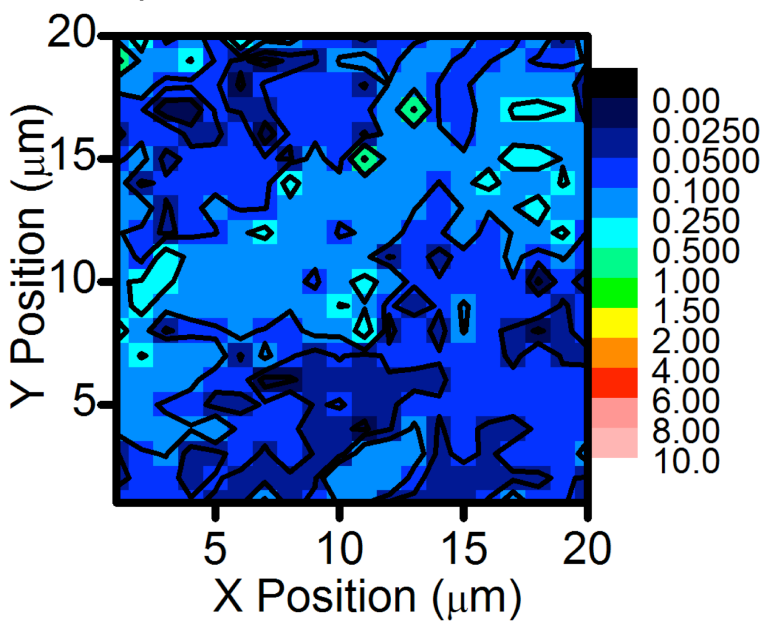

Figure 2. a) Typical raw data for an AFM cantilever deflection unloading curve recorded during a force-displacement measurement. b) Corrected force-displacement plot with 
the applied curve fit of equation (2) used to calculate elastic modulus. c) Distribution map of the elastic modulus with values shown in MPa.

The effects of repeat AFM indentation of an area were conducted by analysis of repeat measurements for seven freshly excised dermal explants. The RMS values for all repeated measurements are shown in Figure 3a. The repeat measurements showed a RMS range between 0.102 and 0.291 , indicating variation in the measurement of the elastic modulus at a single point. Additionally, no discernible change in the measured elastic modulus was observed when indentations were performed in rapid succession at a single point. 

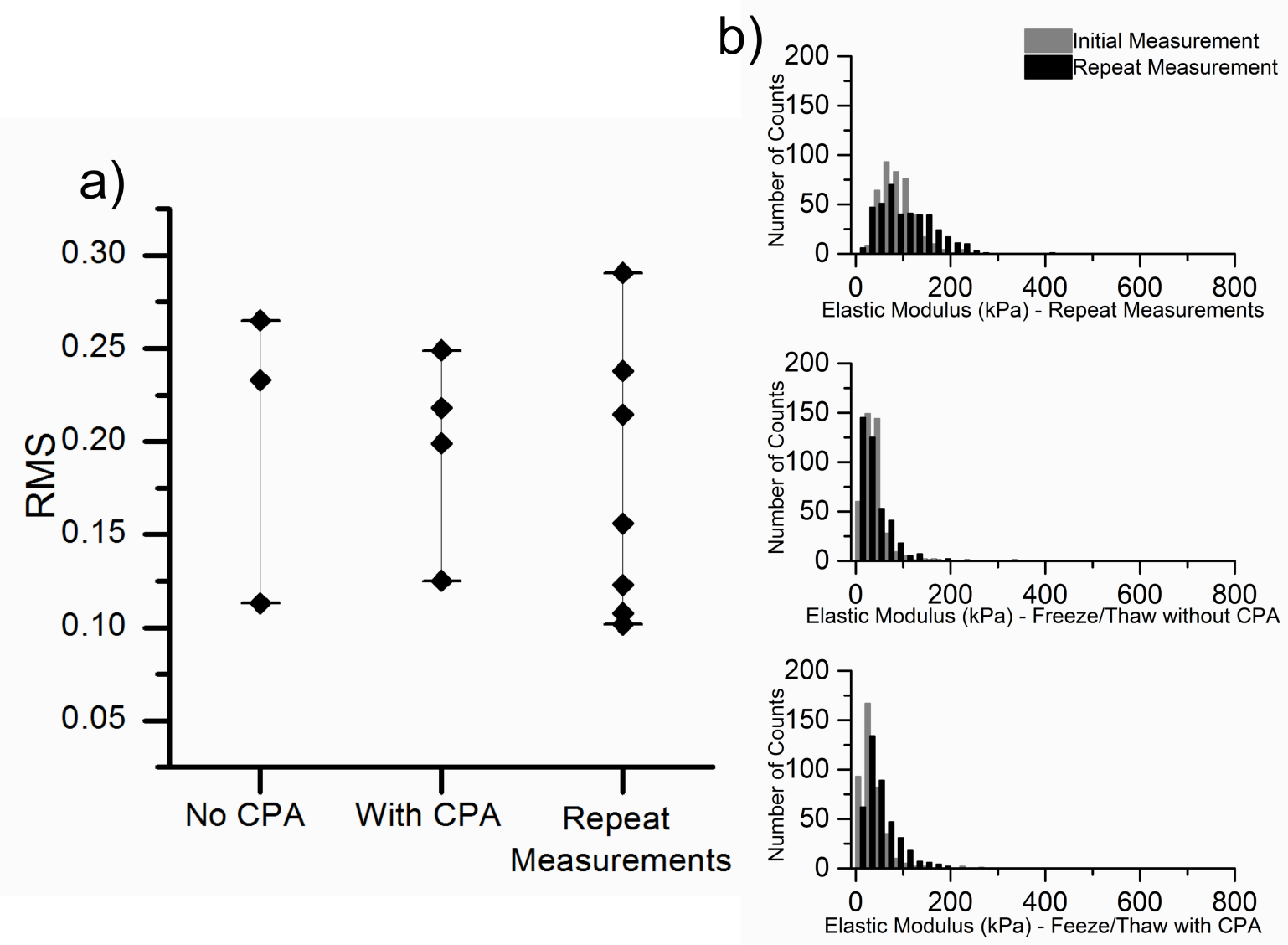

Figure 3. a) Plot of the RMS values for measurements of the elastic modulus before and after the freeze-thaw cycle for untreated, CPA treated samples and repeated measurements on fresh dermal explant samples. b) Histograms of the raw elastic modulus data for each of the sample conditions measured.

The effect of a freezing and thawing cycle on the elastic modulus of the dermal explants was investigated in order to understand potential sample degradation from the preparation methods used. Figure $3 \mathrm{~b}$ displays the histograms of the raw elastic modulus data for each of the conditions. Statistical analysis (D'Agostino-Pearson Omnibus test) indicated that all the data sets were not taken from a normal or 
lognormal distribution. Lognormal distributions were fitted to the data sets to quantify the differences between the data sets. For the repeat measurements, initial location and scale parameters of $\mu=4.464$ and $\sigma=0.3871$ respectively were fitted and the repeat measurement resulted in parameters of $\mu=4.460$ and $\sigma=0.6254$. The sample treated with a CPA initially resulted in parameters of $\mu=3.437$ and $\sigma=0.6941$ with repeat measurements giving $\mu=3.699$ and $\sigma=0.6837$. Fitting to the untreated samples resulted in $\mu=3.916$ and $\sigma=0.4996$ and, after thawing, parameters of $\mu=4.059$ and $\sigma=$ 0.5059. The distributions for each histogram shown in Figure $3 \mathrm{~b}$ are significantly different using the non-parametric paired-sample Wilcoxon signed rank test ( $p$ values of 0.00398 for repeat measurements, $7.49 \mathrm{e}-5$ for the CPA treated sample, and $2.069 \mathrm{e}-9$ for the untreated sample). This statistical analysis and the assigning of distribution parameters provide no conclusive evidence that the freeze/thawing cycle has an effect on the sample mechanics. The use of RMS therefore provides a single parameter that can be used to evaluate the change between data sets. The dermal explants frozen using no CPA resulted in a RMS range between 0.113 and 0.265 while the samples treated with a CPA produced a RMS range between 0.125 and 0.249 . The repeated measurements on samples that had been frozen and thawed produced RMS error values that fall within the range of the initial measurements taken on a fresh sample. This result indicates that there is no distinguishable change in the elastic modulus of the dermis as a result of the freeze thaw cycle when performing measurements at the nanoscale and is comparable to the initial findings shown by Foutz et al. for whole rat skin (Foutz et al., 1992). Performing this analysis confirms that comparisons can be made between the dermis of skin samples prepared by cryosectioning and freshly excised dermal explants. Cryosectioning progressively through the whole skin from the 
dermis to the epidermis and thawing each section to record resultant elastic modulus maps is therefore considered representative of the natural state of skin.

AFM force displacement measurements were performed on transverse skin samples to produce a continuous distribution map of the elastic modulus from the epidermis to the dermis. A representative elastic modulus map for a cryosection is shown in Figure 4a with a representative area on an H\&E stained cryosection shown in Figure 4b. A change in the elastic modulus is clearly observed by examining the position of the indentations from the dermis towards the epidermis. 


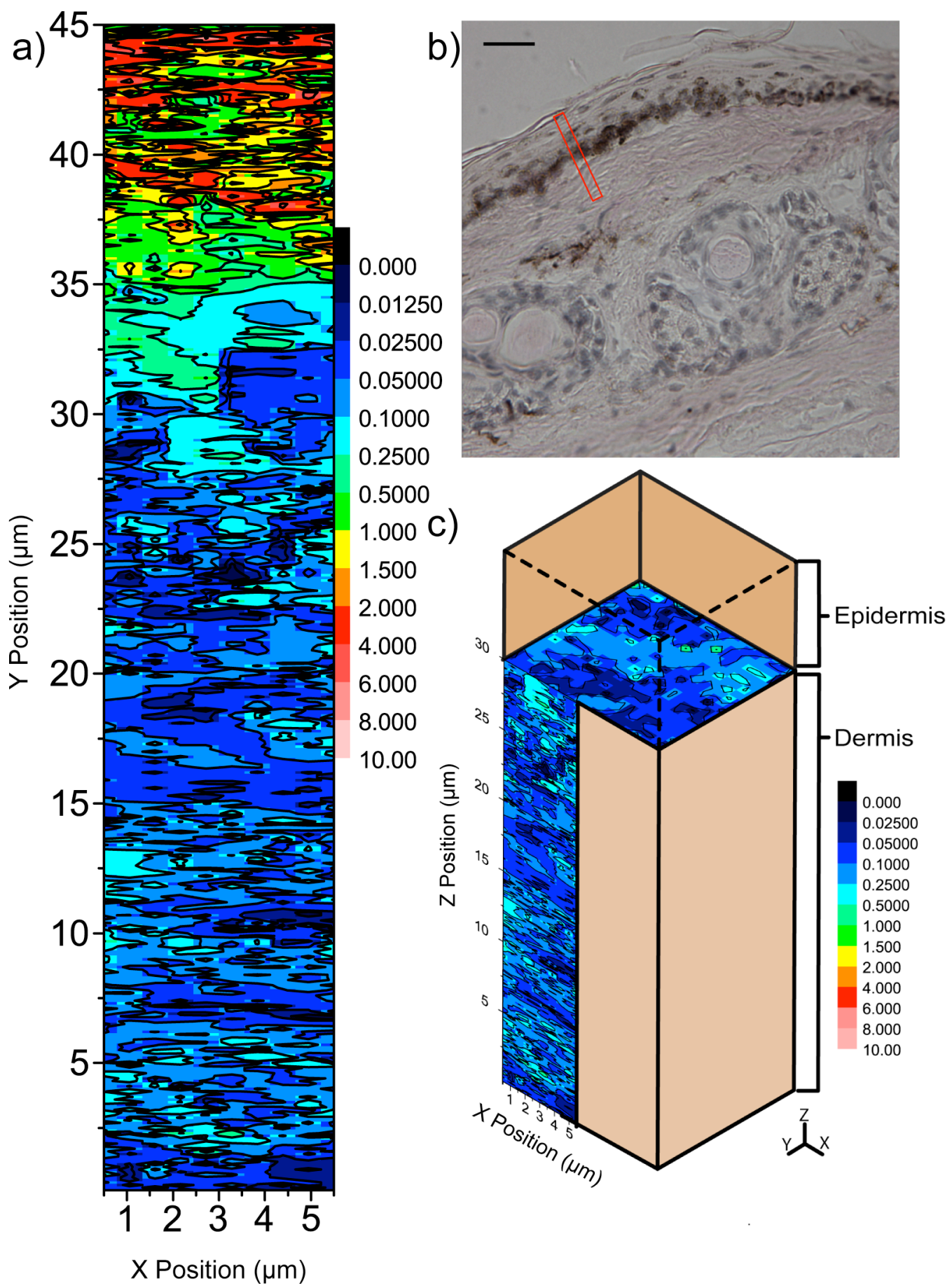

Figure 4. a) Raw elastic modulus distribution of map for through thickness transverse cryosections of mouse tail skin from the epidermis (top) to the dermis (bottom), with values shown in MPa. b) Haematoxylin and eosin staining of cryosection of mouse tail skin with the red box showing a representative area probed by AFM indentation, scale bar $25 \mu \mathrm{m}$. c) Combined representation for the distribution maps of the elastic modulus 
of mouse tail skin. The similar distribution of the elastic modulus when the direction of the applied indentations is changed indicates isotropic behaviour. Modulus values are shown in MPa.

The distribution map of the spatial coordinates and measured elastic modulus shows an almost 100 fold increase in the elastic modulus at the surface of the epidermis when compared to the dermis. The elastic modulus values for the dermis generally fall within the kilopascal range where the epidermal elastic modulus tends towards values in the megapascal range. The elastic modulus values of the dermis for these transverse cryosections were taken from the force displacement measurements of the area at least $20 \mu \mathrm{m}$ from the edge of the sample to eliminate the possibility of the contribution of the epidermis. The elastic modulus value measured for the dermal region ranged from $0.0204 \mathrm{kPa}$ to $598 \mathrm{kPa}$ with a mean value of $60.9 \pm 0.721$ (SEM) $\mathrm{kPa}$. Combining the indentation data from the cryosections and constructing a histogram displays a median value of $44.8 \mathrm{kPa}$, as shown in Figure 5, which displays the raw elastic modulus values. The elastic modulus of the transverse cryosection of the dermis was comparable to the elastic modulus of the dermal surface. For the transverse cryosections the applied indentation load was orthogonal to the loading condition used for the measurement of the dermal explant surface.

The elastic modulus map of the region spanning the dermis and epidermis, Figure 4a, shows the clear change in the mechanics of the tissue at the interface of the dermis and the epidermis. Combining the data sets of the transverse cross section and the dermal explant surface allows for a composite 3D mechanical interpretation of the spatial elastic modulus to be produced, as shown in Figure 4c. The 3D construction of the AFM 
indentation data for the upper dermal region suggests, through the comparison of the elastic modulus values when the sample orientation has been changed relative to the applied load, that the dermis of the mouse tail exhibits an isotropic response to AFM indentation forces. The isotropic response of the dermis suggests that the AFM nanoindentation is not directly indenting the individual components of the dermis and the indentation forces are not sufficient to strain the collagen network. The collagen fibres within the dermis have been shown to be preferentially aligned in one direction, which leads to an anisotropic response at the macroscale (Ní Annaidh et al., 2012a). The collagen fibre network does not influence the mechanical behaviour of the small volume being tested by AFM nanoindentation because the length scale being observed is operating beneath the macroscale where the collagen fibres contribute to the mechanical response. Therefore, application of nanoscale deformations within the dermis suggests a mechanical response that is dominated by the ground substance within the dermis rather than the collagen fibre network. The resulting 3D isotropy of the dermis potentially indicates that, at a cellular level where the cells are applying small forces to the environment, the response of the tissue is independent of the direction of the applied force. The AFM indentation elastic modulus values for the dermal explants and dermal regions of the cryosections were aggregated and a histogram of the frequency of elastic modulus was constructed. As Figure 5a-b shows, both the data for the dermal explants and the cryosections have comparable peaks in the frequency of the elastic modulus in the $40 \mathrm{kPa}$ region. Statistical analysis of the histograms indicates that they distributions are neither normal nor lognormal (D'Agostino-Pearson Omnibus test). However, fitting lognormal distributions to the dermal explant data set results in most likely estimate parameters of $\mu=3.873$ and $\sigma=$ 1.337 leading to a most likely value for the distribution of $48.09 \mathrm{kPa}$. For the 
cryosection, the lognormal most likely estimate parameters are $\mu=3.822$ and $\sigma=$ 0.7670 , which provides a most likely value of $45.69 \mathrm{kPa}$ for the cryosection data. Comparing the two histograms provided no significant difference in the medians $(p=$ 0.747 using the Mann-Whitney U-test) and further indicates mechanical isotropy of the dermis at the nanoscale. 

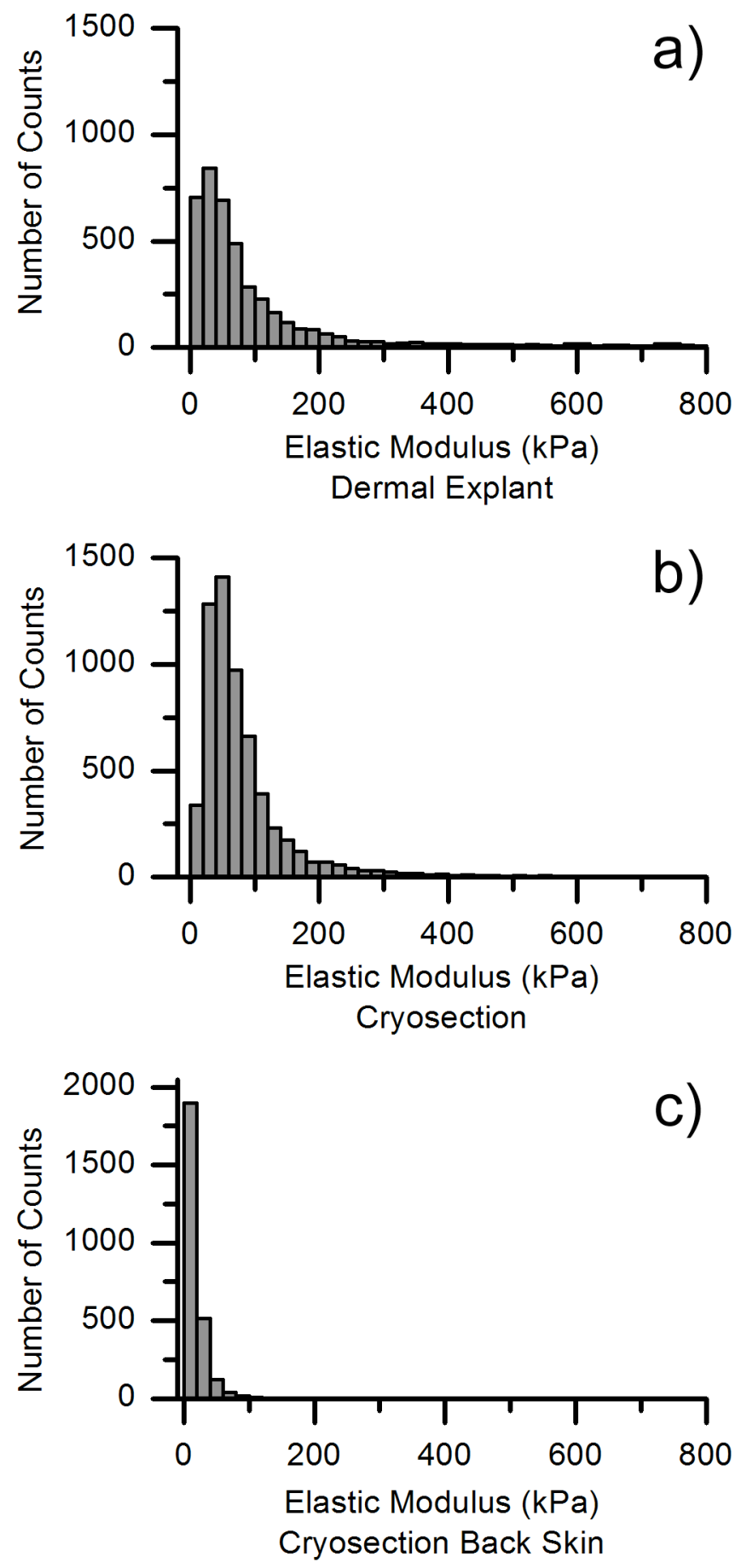

Figure 5. Histograms of the raw elastic modulus data for a variety of samples. a) Dermal explant samples of mouse tail skin b) dermal region of transverse cryosections of mouse tail skin and c) transverse cyrosection of mouse back skin. 
Cryosections of mouse dorsal skin were also taken and analysed using AFM indentations to measure the elastic modulus of the dermal region on transverse cryosections. The AFM indentation results were used to construct a histogram of elastic modulus, shown in Figure 5c, to compare the elastic modulus of the mouse dermis at a various anatomical locations. The distribution had a range of elastic modulus values from a minimum of $0.122 \mathrm{kPa}$ to a maximum value of $167 \mathrm{kPa}$ with a mean value of 13.2 $\pm 0.256(\mathrm{SEM}) \mathrm{kPa}$, and the median data set value of $9.65 \mathrm{kPa}$. The results of the elastic modulus evaluations show a reduction in the measured value for the dorsal skin when compared to the tail when measured along the same plane. Comparing the dorsal skin data set to the cryosection taken from the tail skin resulted in a p value of 0 using the Mann-Whitney U-test indicating that the distributions are statistically different. This result demonstrates an anatomical location dependent elastic modulus for murine skin, but also that the AFM indentation analysis is sensitive enough to evaluate variations in elastic modulus within the same tissue type.

\section{Conclusions}

Spatial mapping of the elastic modulus of mouse tail dermis prepared by a cryosectioning method was performed using AFM indentation. The mechanical response of the mouse dermis was recorded in two directions and the calculated elastic modulus values were found to be independent of loading direction. Our results suggest that the cryosectioning preparation does not have a measureable effect on the elastic modulus of the dermis when measured by AFM indentation and that the dermis acts as an isotropic material in response to an indentation loading condition. This result suggests that cells within the dermis potentially experience an isotropic mechanical 
microenvironment. We also display the positional changes in the elastic modulus of whole mouse skin from the epidermis through to the upper dermis. The AFM indentation results show significant variations in the mechanics of skin between the different layers of skin. Measurements conducted on various anatomical regions of skin showed significant differences in the elastic modulus, demonstrating the need for further investigations into the changes in the physiological environment that lead to the variation in mechanical properties at the nanoscale. Such conclusions indicate the potential for exploring the structural role of the constituent layers of skin and contribution of each layer to the mechanical response of whole skin.

\section{Acknowledgements}

A. K. thanks Queen Mary University of London for scholarship funding. The authors would like to thank Dr. Kristin Braun for providing the mice and her assistance with the tissue processing, and the NanoVision Centre at Queen Mary University of London for use of AFM facilities. 


\section{References}

Achterberg, V.F., Buscemi, L., Diekmann, H., Smith-Clerc, J., Schwengler, H., Meister, J.-J., Wenck, H., Gallinat, S., Hinz, B., 2014. The nano-scale mechanical properties of the extracellular matrix regulate dermal fibroblast function. J. Invest. Dermatol. 134, 1862-72. doi:10.1038/jid.2014.90

Bakhach, J., 2009. The cryopreservation of composite tissues: Principles and recent advancement on cryopreservation of different type of tissues. Organogenesis 5, 119-126. doi:10.4161/org.5.3.9583

Braun, K.M., Niemann, C., Jensen, U.B., Sundberg, J.P., Silva-Vargas, V., Watt, F.M., 2003. Manipulation of stem cell proliferation and lineage commitment: visualisation of label-retaining cells in wholemounts of mouse epidermis. Development 130, 52415255. doi:10.1242/dev.00703

Bravo, D., Rigley, T.H., Gibran, N., Strong, D.M., Newman-Gage, H., 2000. Effect of storage and preservation methods on viability in transplantable human skin allografts. Burns 26, 367-378.

Butt, H., Jaschke, M., 1995. Calculation of thermal noise in atomic force microscopy. Nanotechnology 6, 1-7.

Chen, F., Zhang, W., Wu, W., Jin, Y., Cen, L., Kretlow, J.D., Gao, W., Dai, Z., Wang, J., Zhou, G., Liu, W., Cui, L., Cao, Y., 2011. Cryopreservation of tissue-engineered epithelial sheets in trehalose. Biomaterials 32, 8426-8435.

Crichton, M.L., Chen, X., Huang, H., Kendall, M.A.F., 2013. Elastic modulus and viscoelastic properties of full thickness skin characterised at micro scales. Biomaterials 34, 2087-2097.

Crichton, M.L., Donose, B.C., Chen, X., Raphael, A.P., Huang, H., Kendall, M.A.F., 2011. The viscoelastic, hyperelastic and scale dependent behaviour of freshly excised individual skin layers. Biomaterials 32, 4670-4681.

Delalleau, A., Josse, G., Lagarde, J.-M., Zahouani, H., Bergheau, J.-M., 2006.

Characterization of the mechanical properties of skin by inverse analysis combined with the indentation test. J. Biomech. 39, 1603-10.

doi:10.1016/j.jbiomech.2005.05.001

Diridollou, S., Patat, F., Gens, F., Vaillant, L., Black, D., Lagarde, J.M., Gall, Y., Berson, M., 2000. In vivo model of the mechanical properties of the human skin under suction. Ski. Res. Technol. 6, 214-221.

Domke, J., Radmacher, M., 1998. Measuring the elastic properties of thin polymer films with the atomic force microscope. Langmuir 14, 3320-3325. 
Engler, A.J., Sen, S., Sweeney, H.L., Discher, D.E., 2006. Matrix elasticity directs stem cell lineage specification. Cell 126, 677-89. doi:10.1016/j.cell.2006.06.044

Foutz, T.L., Stone, E.A., Abrams, C.F., 1992. Effects of Freezing on Mechanical - Properties of Rat Skin. Am. J. Vet. Resarch 53, 788-792.

Fuller, B.J., 2004. Cryoprotectants: the essential antifreezes to protect life in the frozen state. Cryo Letters 25, 375-88.

Gahagnon, S., Mofid, Y., Josse, G., Ossant, F., 2012. Skin anisotropy in vivo and initial natural stress effect: a quantitative study using high-frequency static elastography. J. Biomech. 45, 2860-5. doi:10.1016/j.jbiomech.2012.08.032

Gąsior-Głogowska, M., Komorowska, M., Hanuza, J., Mączka, M., Zając, a, Ptak, M., Będziński, R., Kobielarz, M., Maksymowicz, K., Kuropka, P., Szotek, S., 2013. FTRaman spectroscopic study of human skin subjected to uniaxial stress. J. Mech. Behav. Biomed. Mater. 18, 240-52. doi:10.1016/j.jmbbm.2012.11.023

Gavara, N., Chadwick, R.S., 2012. Determination of the elastic moduli of thin samples and adherent cells using conical atomic force microscope tips. Nat. Nanotechnol. 7, 733-6. doi:10.1038/nnano.2012.163

Geerligs, M., van Breemen, L., Peters, G., Ackermans, P., Baaijens, F., Oomens, C., 2011. In vitro indentation to determine the mechanical properties of epidermis. J. Biomech. 44, 1176-1181.

Gerhardt, L.C., Schmidt, J., Sanz-Herrera, J. a., Baaijens, F.P.T., Ansari, T., Peters, G.W.M., Oomens, C.W.J., 2012. A novel method for visualising and quantifying throughplane skin layer deformations. J. Mech. Behav. Biomed. Mater. 14, 199-207. doi:10.1016/j.jmbbm.2012.05.014

Grant, C.A., Twigg, P.C., Tobin, D.J., 2012. Static and dynamic nanomechanical properties of human skin tissue using atomic force microscopy: effect of scarring in the upper dermis. Acta Biomater. 8, 4123-9. doi:10.1016/j.actbio.2012.06.042

Grover, C.N., Cameron, R.E., Best, S.M., 2012. Investigating the morphological, mechanical and degradation properties of scaffolds comprising collagen, gelatin and elastin for use in soft tissue engineering. J. Mech. Behav. Biomed. Mater. 10, 6274. doi:10.1016/j.jmbbm.2012.02.028

Groves, R.B., Coulman, S. a, Birchall, J.C., Evans, S.L., 2013. An anisotropic, hyperelastic model for skin: experimental measurements, finite element modelling and identification of parameters for human and murine skin. J. Mech. Behav. Biomed. Mater. 18, 167-80. doi:10.1016/j.jmbbm.2012.10.021

Hang, F., Barber, A.H., 2011. Nano-mechanical properties of individual mineralized collagen fibrils from bone tissue. J. R. Soc. Interface 8, 500-505.

doi:10.1098/rsif.2010.0413 
Hang, F., Gupta, H.S., Barber, A.H., 2014. Nanointerfacial strength between noncollagenous protein and collagen fibrils in antler bone. J. R. Soc. Interface 11, 20130993. doi:10.1098/rsif.2013.0993

Heim, A.J., Matthews, W.G., Koob, T.J., 2006. Determination of the elastic modulus of native collagen fibrils via radial indentation. Appl. Phys. Lett. 89, 181902. doi:10.1063/1.2367660

Hendriks, F.M., Brokken, D., van Eemeren, J., Oomens, C.W.J., Baaijens, F.P.T., Horsten, J., 2003. A numerical-experimental method to characterize the non-linear mechanical behaviour of human skin. Ski. Res. Technol. 9, 274-283.

Jachowicz, J., McMullen, R., Prettypaul, D., 2007. Indentometric analysis of in vivo skin and comparison with artificial skin models. Ski. Res. Technol. 13, 299-309. doi:10.1111/j.1600-0846.2007.00229.x

Jee, T., Komvopoulos, K., 2014. In vitro measurement of the mechanical properties of skin by nano/microindentation methods. J. Biomech. 47, 1186-92. doi:10.1016/j.jbiomech.2013.10.020

Last, J.A., Liliensiek, S.J., Nealey, P.F., Murphy, C.J., 2009. Determining the mechanical properties of human corneal basement membranes with atomic force microscopy. J. Struct. Biol. 167, 19-24.

Lin, D.C., Dimitriadis, E.K., Horkay, F., 2007. Robust strategies for automated AFM force curve analysis - I. Non-adhesive indentation of soft, inhomogeneous materials. J. Biomech. Eng. Asme 129, 430-440. doi:10.1115/1.2720924

Lo, C.M., Wang, H.B., Dembo, M., Wang, Y.L., 2000. Cell movement is guided by the rigidity of the substrate. Biophys. J. 79, 144-52. doi:10.1016/S00063495(00)76279-5

Ní Annaidh, A., Bruyère, K., Destrade, M., Gilchrist, M.D., Maurini, C., Otténio, M., Saccomandi, G., 2012a. Automated estimation of collagen fibre dispersion in the dermis and its contribution to the anisotropic behaviour of skin. Ann. Biomed. Eng. 40, 1666-78. doi:10.1007/s10439-012-0542-3

Ní Annaidh, A., Bruyère, K., Destrade, M., Gilchrist, M.D., Otténio, M., 2012b. Characterization of the anisotropic mechanical properties of excised human skin. J. Mech. Behav. Biomed. Mater. 5, 139-48. doi:10.1016/j.jmbbm.2011.08.016

Osawa, T., Onodera, M., Feng, X.Y., Nozaka, Y., 2003. Comparison of the thickness of basement membranes in various tissues of the rat. J. Electron Microsc. (Tokyo). 52, 435-440.

Ottenio, M., Tran, D., Ní Annaidh, A., Gilchrist, M.D., Bruyère, K., 2014. Strain rate and anisotropy effects on the tensile failure characteristics of human skin. J. Mech. Behav. Biomed. Mater. doi:10.1016/j.jmbbm.2014.10.006 
Petrie, R.J., Gavara, N., Chadwick, R.S., Yamada, K.M., 2012. Nonpolarized signaling reveals two distinct modes of 3D cell migration. J. Cell Biol. 197, 439-55. doi:10.1083/jcb.201201124

Radmacher, M., 1997. Measuring the elastic properties of biological samples with the AFM. Ieee Eng. Med. Biol. Mag. 16, 47-57.

Rehfeldt, F., Engler, A.J., Eckhardt, A., Ahmed, F., Discher, D.E., 2007. Cell responses to the mechanochemical microenvironment - Implications for regenerative medicine and drug delivery. Adv. Drug Deliv. Rev. 59, 1329-1339.

Saez, A., Buguin, A., Silberzan, P., Ladoux, B., 2005. Is the mechanical activity of epithelial cells controlled by deformations or forces? Biophys. J. 89, L52-4. doi:10.1529/biophysj.105.071217

Silver, F.H., Freeman, J.W., DeVore, D., 2001. Viscoelastic properties of human skin and processed dermis. Ski. Res. Technol. 7, 18-23.

Silver, F.H., Siperko, L.M., Seehra, G.P., 2003. Mechanobiology of force transduction in dermal tissue. Ski. Res. Technol. 9, 3-23.

Sneddon, I.N., 1948. Boussinesq's problem for a rigid cone. Math. Proc. Cambridge Philos. Soc. 44, 492-507. doi:10.1093/qmath/os-10.1.161

Solon, J., Levental, I., Sengupta, K., Georges, P.C., Janmey, P. a, 2007. Fibroblast adaptation and stiffness matching to soft elastic substrates. Biophys. J. 93, 4453-61. doi:10.1529/biophysj.106.101386

Tsui, T.Y., Pharr, G.M., 1999. Substrate effects on nanoindentation mechanical property measurement of soft films on hard substrates. J. Mater. Res. 14, 292-301. doi:10.1557/JMR.1999.0042

Wang, X., Li, Q., Hu, X., Ma, L., You, C., Zheng, Y., Sun, H., Han, C., Gao, C., 2012. Fabrication and characterization of poly(l-lactide-co-glycolide) knitted mesh-reinforced collagen-chitosan hybrid scaffolds for dermal tissue engineering. J. Mech. Behav. Biomed. Mater. 8, 204-215. doi:10.1016/j.jmbbm.2012.01.001

Wang, Y., Marshall, K.L., Baba, Y., Gerling, G.J., Lumpkin, E. a, 2013. Hyperelastic Material Properties of Mouse Skin under Compression. PLoS One 8, e67439. doi:10.1371/journal.pone.0067439

Yuan, Y.H., Verma, R., 2006. Measuring microelastic properties of stratum corneum. Colloids and Surfaces B-Biointerfaces 48, 6-12.

Zahouani, H., Pailler-Mattei, C., Sohm, B., Vargiolu, R., Cenizo, V., Debret, R., 2009. Characterization of the mechanical properties of a dermal equivalent compared with human skin in vivo by indentation and static friction tests. Ski. Res. Technol. 15, 68-76. doi:10.1111/j.1600-0846.2008.00329.x 


\section{Supplementary Information}

\section{Calibration of AFM cantilever deflection}

Force-displacement measurements were conducted on sapphire sheets, which have a known elastic modulus of $345 \mathrm{GPa}$ and are used to measure the ratio of photodiode current (nA) to physical cantilever deflection (nm). Supplementary Figure 1a shows a typical force-displacement curve conducted on a sapphire sheet displaying the linear relationship between the cantilever deflection and the z-piezo displacement. Supplementary Figure 1b shows the unloading portion of a force-displacement curve conducted within the epidermis. The force-displacement curve displays non-linear behaviour indicating that the increased modulus of the epidermis compared to the dermis is not an artefact. 


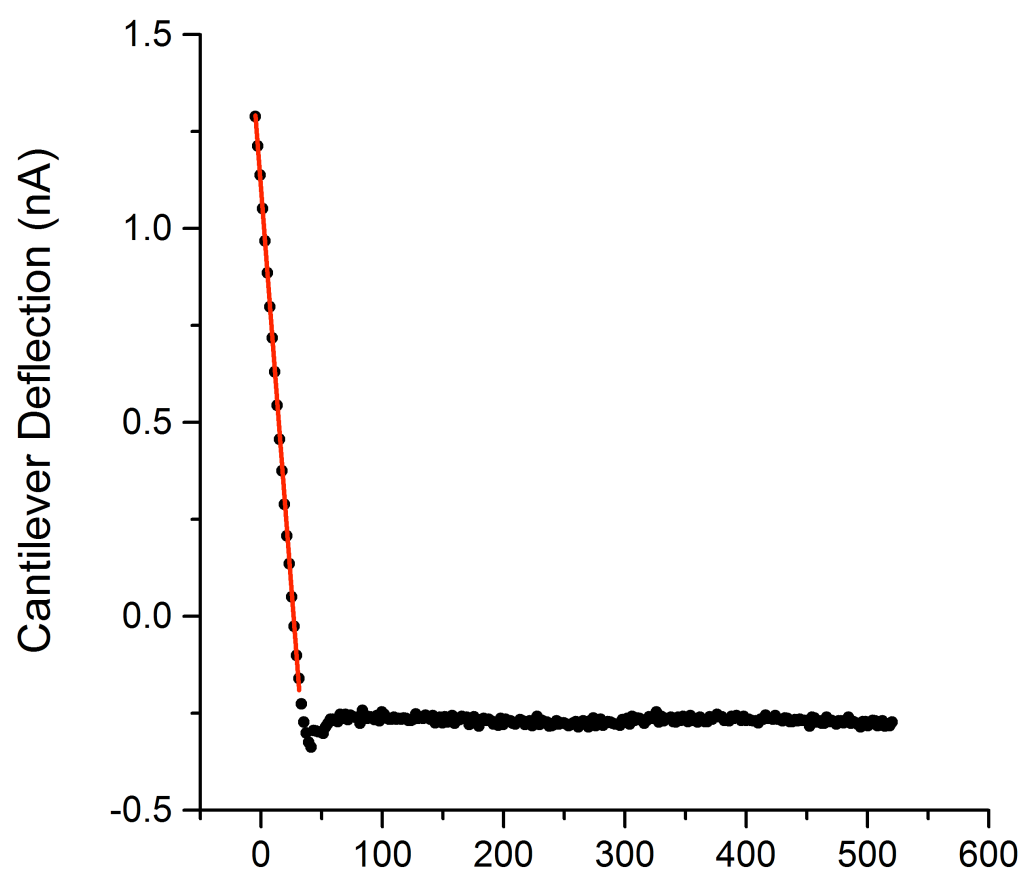

Z-Piezo Displacement (nm) - Calibration Curve

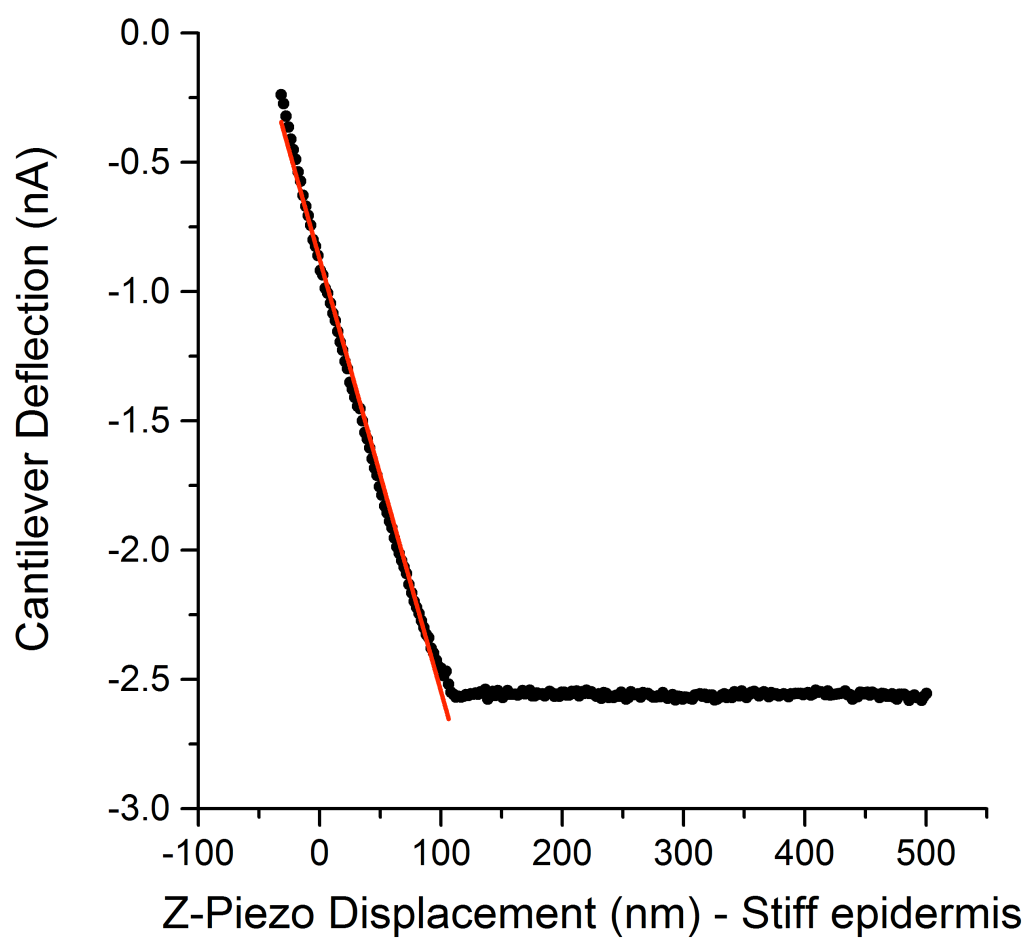


Supplementary Figure 1. (top) force-displacement curve conducted on a sapphire sheet showing linear relationship between the cantilever deflection and z-piezo displacement. (bottom) force-displacement curve from indentation of the epidermis showing the nonlinear indentation behaviour.

Validation of AFM indentation measurements

Agarose gels were produced at two different weight percent compositions by dissolving the dry agarose in PBS overnight at $70^{\circ} \mathrm{C}$ and casting the gels at room temperature in tissue culture plastic petrie dishes. Gels were then stored at $4{ }^{\circ} \mathrm{C}$ overnight in PBS prior to testing. 50 force-displacement measurements were conducted on each gel and the elastic modulus was calculated. The results of the elastic modulus show good agreement with the elastic modulus of the same gels when measured by unconfined compression. Additionally, a significant difference $(p<0.001)$ between the elastic modulus of the two gels when measured by AFM indentation was observed, indicating the AFM indentation results are both accurate and precise. 


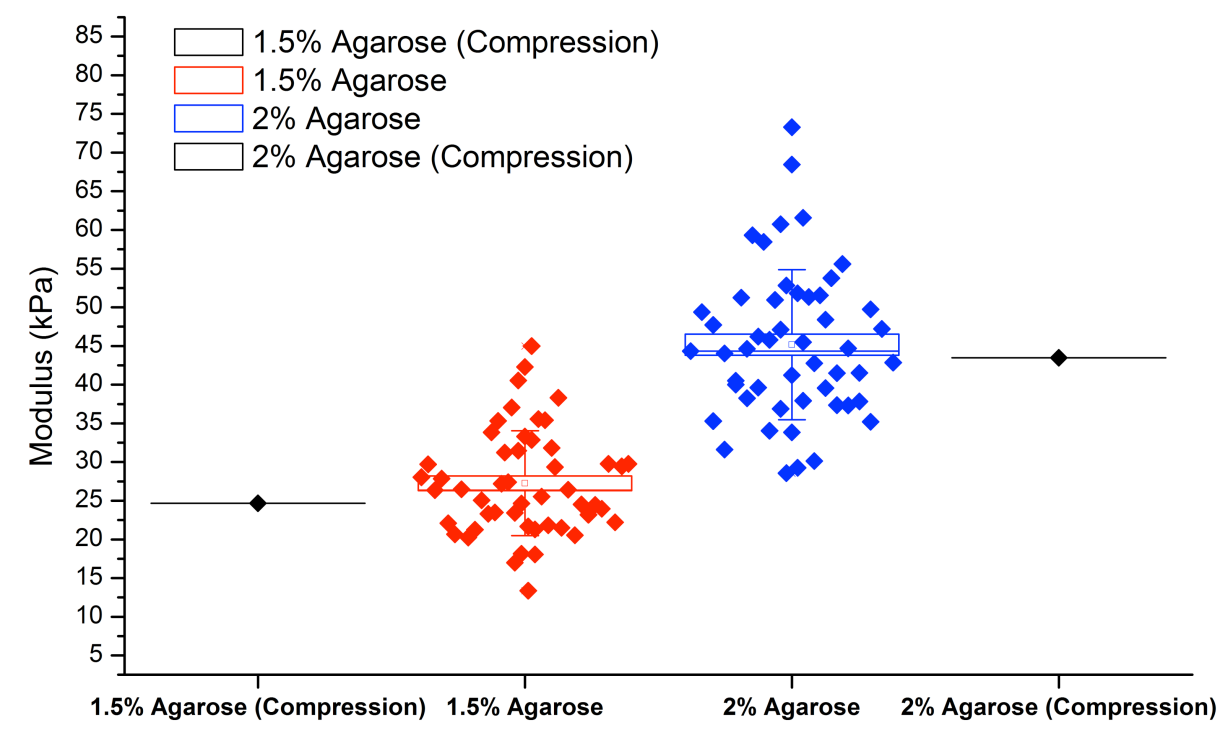

Supplementary Figure 2. Elastic modulus values for agarose gels. Box represents standard error of mean and the whiskers show the standard deviation. Significant difference in the elastic modulus between the $1.5 \%$ and the $2 \%$ agarose at the $p<0.001$ level is observed. Compression values were independently determined using the same agarose gels. 